\title{
Towards LBL Positioning Systems for Multiple Vehicles
}

\author{
José Melo, Aníbal Matos \\ Faculty of Engineering, University of Porto \\ and \\ INESC TEC \\ \{jose.melo,anibal\}@fe.up.pt
}

\begin{abstract}
In this article we discuss the use of LBL acoustic networks for operations with multiple AUVs. Differently from standard LBL configurations, we propose to use the One-WayTravel-Time of acoustic signals to compute the ranges between all the devices. Moreover, we derive the suitable algorithms for both the navigation of multiple vehicles, but also their external tracking. Experimental results are provided that support the evidence that our approach is successful in operations for multiple vehicles.
\end{abstract}

\section{INTRODUCTION}

The main navigation solution for current Autonomous Underwater Vehicles (AUVs) is a combination of both inertial and acoustic navigation techniques. Inertial Navigation alone is known to produce position estimates that will drift and grow unbounded over time. Bounding the errors of inertial navigation requires the use of some kind of navigational aids. While other methods exist, the most common way for AUVs to acquire this position navigation aids is by resorting to Acoustic Navigation. Acoustic Navigation methods rely on the exchange of the acoustic signals between the nodes of an acoustic network. The use of such techniques is able to provide navigation aids to the vehicles, but they also give the possibility for externally tracking the trajectory of the vehicles. This functionality, that allows the real-time monitoring of the operations, can be of extreme importance, particularly in the more demanding and dangerous applications, like in the oil or military industries. Nevertheless, most of these methods have been envisioned for the single-vehicle scenario and don't have an obvious extension to the multiple vehicle case. For a more thorough review on recent AUV Navigation and Localization techniques, refer to [1].

AUVs are already a reliable and cost-effective solution to autonomously perform a range of underwater tasks. Examples for this can be, among others, bathymetric tasks, environmental surveying or even mine countermeasures operations. However, the efficiency of such operations could be even higher if multiple cooperating vehicles are used. In this article we propose an Acoustic Network scheme that is able to address the problem of simultaneous navigation and external tracking of multiple AUVs, nowadays a very relevant topic. This approach is composed of both the hardware and the software necessary to implement the desired acoustic network. Our proposal is an extension of traditional LBL methods, and does not need any communication link between the vehicles or the beacons. Up to the authors knowledge, it is the first time that a similar system is proposed. The organization of this article is as follows. Section II provides background information on relevant work found in the literature. Then, in Section III we describe the Acoustic Network proposed and details of its implementation. After that, in Section IV we introduce a generic navigation filter to be implemented in the AUVs, and we continue with detailing the algorithm that is able to simultaneously track all the vehicles in operation in Section V. Furthermore, an experimental validation of obtained results is provided in section VI and, finally, in Section VII we present some conclusions and discussion about the proposed approach, and point out future research directions.

\section{BACKGROUND}

Acoustic Navigation embraces a number of techniques that rely on the computation of ranges between different acoustic sources, usually a vehicle and a series of acoustic beacons. Acoustic Navigation systems are generally classified according to the size of the baseline, which is the distance between each of the acoustic beacons. Accordingly, Long Baseline (LBL), Short Baseline (SBL) and Ultra-Short Baseline (USBL) are the most common configurations used. While in LBL methods the beacons are widely spaced and stationary, deployed whether at the surface or at the bottom of the sea, in both SBL and USBL methods the beacons are usually mounted on the hull of a ship. If the velocity of propagation of a sound wave in the water is locally known, the Time-of-Flight (TOF) of an acoustic signal is used to obtain slant ranges between the vehicle and the set of acoustic beacons. From there, the position of the vehicle can be easily estimated. Additionally, in SBL or USBL configurations, sometimes bearing can also be computed by comparing, respectively, the differences in the "time-of-arrival" or phase of the incoming signal as detected by each of the acoustic beacons.

LBL systems are one of the most robust, reliable and accurate configurations of Acoustic Navigation systems. Moreover they can easily be used for external tracking, by eavesdropping the acoustic signals exchanged between the beacons and the AUVs. For those reasons, on the remainder of this article particular attention will be given to these type of systems. However a similar reasoning can be used when resorting 
to other type of systems. In specific the focus will be on using GPS enabled intelligent buoys as acoustic beacons, implementing a scheme that is also known as Inverted LBL. Compared to traditional LBL systems, on which the beacons are deployed at the bottom of the sea, using such buoys is more convenient as the beacons remain in the surface and their exact position can be easily retrieved with the help of GPS.

In LBL systems, the location of each one of the beacon needs to be determined previously to operation time. Then, during the missions, the vehicle interrogates the beacons acoustically and measures the round-trip travel-time to each beacon. The time difference between sending the query and receiving the reply, also known as Two Way Travel Time (TWTT), is then converted to the slant range between AUV and a given beacon. The signals emitted by each one of the beacons must be uniquely identifiable, which is typically accomplished through the assignment of unique frequency modulated signals to each beacon. LBL algorithms provide navigational aids to an AUV in terms of ranges to each one of the beacons that compose the network, which then constitute spherical navigation constraints to the general problem of relative positioning of the vehicle. Externally tracking an AUV is a feature that is often sought when dealing with Acoustic Navigation systems, as it allows real-time monitoring of the position of the vehicle. This can be achieved by eavesdropping the acoustic signals exchanged in the network and, similarly to the navigation case, using appropriate multilateration techniques to compute the position of the vehicle using the ranges to the different beacons. An example of such feature can be found in [2].

\section{A. Operations with Multiple Vehicles}

Scaling up LBL systems for addressing the navigation and external tracking of multiple vehicles has no easy and standard solution. On one hand it is desirable that AUVs keep receiving position updates from the acoustic beacons at least at the same rate. That means that ingenuous approaches, relying on Time Division Multiple Access (TDMA) like schemes, assigning time slots to each one of the vehicles to interrogate be beacons is not desirable. As the update rate for each vehicle decreases with the number of vehicles present, this would lead to undesirable degradation of the performance of the system. In practice, this limits LBL navigation of multiple vehicles to only very few vehicles at most. On the other hand the external tracking feature requires that the vehicles need to be active, that is, the vehicles need to emit acoustic signals. Moreover, each of the beacons also needs to somehow be able to associate each vehicle with the signals it has emitted.

A naive approach for this problem was suggested in [3], on which the authors propose to have different vehicles emitting distinguishable acoustic signals, by using signals modulated with different frequencies. While this approach works, in the sense that it allows both the navigation and tracking of multiple vehicles, increasing the number of vehicles has a direct impact in terms of complexity of the acoustic system, its development and tuning, but also on its cost. An alternative approach for the problem of simultaneous navigation of multiple AUVs was presented in [4]. There, the authors described a method suitable to a fleet of AUVs, on which a leader vehicle is navigating conventionally with an LBL network. Other vehicles of the fleet should be able to intercept this signals, but also to obtain their relative angular heading relative to the sources of the signals. With this information it is possible for all the vehicles to determine their position relative to their leader. In [5] the authors considered a similar problem, by proposing a fully decentralized localization system that enables the operations of a swarm of AUVs. The approach consisted on the use of the developed localization sensor, capable of producing instantaneous estimates of relative azimuth, range, and heading of neighboring AUVs. It should be noted however that such approach provides only relative position estimates, and requires that all the vehicles to be in close proximity to each other. Recent advances in underwater acoustic communications have also brought acoustic modems to play a relevant part in underwater navigation capabilities. In fact several authors have proposed different frameworks to enable Cooperative Navigation, for example [6]. In Cooperative Navigation, teams of AUVs localize themselves more accurately by sharing position estimates and uncertainty. However, such approaches require a data link between the vehicles. While this is a interesting approach, acoustic communications are still characterized by small bandwidth, low data rates and high latency and, particularly for shallow waters and adverse environmental conditions, reliable underwater communications can be quite challenging for long distances.

Traditional LBL systems are based on measuring the TwoWay-Travel-Time (TWTT) of an acoustic signal, on which a vehicle interrogates a set of beacon and waits for their reply. This has obvious disadvantages when using multiple vehicles. In order to overcome this fundamental issue of $\mathrm{LBL}$ systems, it has been proposed to use systems based on the OneWay-Travel-Time of acoustic signals. In this kind of systems, instead of having the vehicle interrogating the beacons, the beacons broadcast their acoustic signals at time instants that are know by all the devices operating. Such systems have the obvious advantage of being applicable to the navigation of multiple vehicles. Perhaps one of the first proposals for OWTT LBL navigation systems was discussed in [7]. There, the development of a synchronous-clock acoustic communication/navigation system for underwater vehicles that would enable navigation for fleet of AUVs was discussed. Then, other articles followed focused on the estimation framework for the vehicles that would be able to fuse LBL OWTT ranges with data from other navigation sensors [8], [9], [10]. The main drawback initially pointed out to OWTT based systems was the requirement for the clocks of all the devices, beacons and vehicles, to be synchronized. Because the OWTT measurement is based on the difference between the sender's emission time and reception time on the receiver, it is crucial that the clocks of all the nodes that constitute the network are synchronized to within an acceptable tolerance. While initially OWTT systems meant increased complexity in terms 
of hardware design of the clock sources, but also in terms on synchronization algorithms, nowadays there are already the technological solutions that can cope, at least partially, with such issues in a fairly straightforward way. Moreover, filtering algorithms have emerged that estimate the unknown offset between different clock sources, thus overcoming an explicit synchronization between vehicles and beacons [11]. Although OWTT techniques do in fact enable the navigation of multiple vehicles, they fail to address the external tracking problem.

\section{AcOustic NeTwork}

From the different acoustic positioning methods, LBL systems stand out mostly due to their ability to provide absolute coordinates with constant accuracy. However, from the previous section, it is clear that no obvious solution to the problem of navigation and external tracking of multiple AUVs has been envisioned yet for these systems. Traditional LBL positioning systems use the TWTT travel times and convert them to ranges to two or more widely spaced stationary beacons, situated whether on the sea bottom or at the surface. However, TWTT based systems are not easily scalable to cope with multiple vehicles. On the other hand, it has been demonstrated, for example in [8] that systems based on OWTT can easily provide navigational aids to multiple vehicles by broadcasting their signals on the network, but they don't address the external tracking problem. In this sections, we will describe the acoustic network that we propose in order to address this problem, that tries to take advantage of the strengths of the different acoustic navigation methods proposed in the literature. On top of the acoustic network here described, our solution is also dependent on suitable navigation and tracking algorithms, which will be discussed in the following sections.

The configuration of the acoustic network here considered consists on a set of acoustic beacons deployed in a fixed predefined location, whether at the surface or at the bottom. While usually at least 4 different beacons are required for a complete 3-dimensional localization of the vehicles relative to the beacons, under some assumptions fewer beacons are required. In the remainder of this work we will consider a configuration with only 2 beacons, but what follows still holds if more beacons are considered. In order to be able to operate with only this small number of beacons, some premises must be verified. First, the length of the baseline must be long enough when compared to the depths at which the AUVs navigate. Also, vehicles must also be able to independently measure its own depth which relative accuracy. Additionally, we are considering that none of the vehicles is crossing the baseline. With this assumptions, in practice we are reducing the position estimation problem of the different vehicles to the horizontal plane only. For more informations regarding this process, refer to [2] and the references therein.

The network of beacons will provide navigational aids to a fleet of AUVs, that can contain a varying number of vehicles, without any limitation. All the nodes in the network, vehicles or beacons, should have their clock sources synchronized. The idea is that at predefined instants of time all the beacons in the network emit an acoustic signal, to be detected and distinguishable by all the vehicles present. This should be accomplished by having the beacons emitting different frequency modulated signals. Simultaneously, all the vehicles should also emit acoustic signals, but all the vehicles can emit similar ones. Then, a centralized estimation algorithm is used that combines the signal detections of all the beacons, otherwise undistinguishable, and estimates the position of each one of the vehicles. In total, a number of $N+1$ distinguishable acoustic signals is required for this system, where $N$ is the number of beacons in use. It is relevant to note that this number remains constant with the number of vehicles in use. The operation of the network then relies on all the nodes converting the OWTT of the detected acoustic signals, and converting them to ranges. Thus, the system draws insights from hyperbolic positioning schemes. The rate at which all the nodes are requested to be emitting will limit the area of operations. If, for example, this rate is $1 \mathrm{~Hz}$, then all the nodes will only consider ranges of up to $1500 \mathrm{~m}$, considering that the local speed of sound is of $1500 \mathrm{~ms}^{-1}$. Naturally, decreasing this rate allows wider areas of operation but, at the same time, also decreases the rate at which new measurement updates are received by each vehicle. Compared to standard LBL algorithms, OWTT systems halve this interval of time, which is of obvious interest.

As it can be noted from the description above, this network has similarities with traditional LBL implementations, in the sense that different beacons must use distinguishable signals, and also because vehicles are required to be active. On the other hand, this network also borrows from OWTT networks, as the interrogation cycle from LBL is replaced by synchronous emission of signals by all the nodes. However, in order for all of this to work, the following assumptions must hold. First all the nodes remain synchronous during the entire duration of the missions. The second assumption stipulates that a locally homogeneous sound speed profile should be observed in the area of operations, which means that the sound velocity should be constant. While this is a moderately mild and common assumption for acoustic navigation systems, it can be problematic if vehicles are navigating across multiple water layers. Finally, it is also required that the vehicles remain in a confined area, so that they can always be tracked by all the beacons.

\section{A. Acoustic Nodes}

The acoustic nodes of the network will be of two kinds: the acoustic beacons, here considered to be a set of synchronized, GPS enabled intelligent buoys developed in-house, and the vehicles in operation. In terms of hardware, all the nodes are equipped with an underwater transducer, operating with frequencies in the range of $20-30 \mathrm{~Hz}$. The transducer, which allows the emission and detection of the signals, is controlled by a proprietary Acoustic Control System (ACS). On the receiver board, a set of analog filters are tuned to distinguish up to 8 distinct frequencies, allowing for the parallel detection and time-stamping of 8 incoming signals. Moreover, for standard 
operating conditions, range detection precisions in the order of $15 \mathrm{~cm}$ can be achieved. Figure 1 presents the ACS boards. The ACS also has the possibility of being triggered by an external source. This is fundamental for synchronizing the clock sources. For the surface acoustic beacons a synchronization system based on the PPS signal provided by some GPS receives is employed. As for the submerged nodes, a precision low-drift clock source needs to be used. Furthermore, the acoustic beacons can also communicate their detections to a basestation, using whether an RF or WiFi link, where the centralized multiple vehicle tracker will be executed.

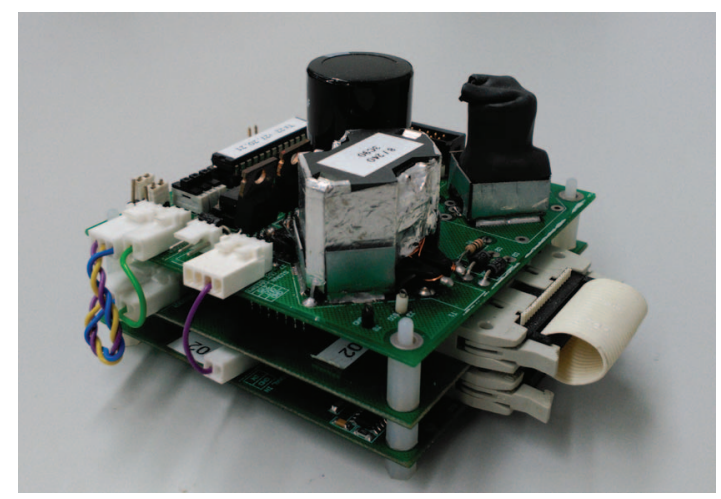

Fig. 1. Acoustic Boards responsible for controlling the emission and detection of the signals

The acoustic boards, mentioned above, are responsible to emit the acoustic signals and precisely time the detection of them. Then, the One-Way-Travel-Time of the acoustic signals have to be converted to ranges, provided that the sound velocity in the area of operations needs is known. However, range observations will naturally be very noisy. Acoustic phenomenons like the multipath of the acoustic signals, caused by the multiple reflections of the signals for example at the bottom, the surface, or even at the margins, are likely to be observed. Because of them, multiple signal receptions will be detected on the receiver side of the ACS. An example of a situation where this phenomenons occur can be seen in Figure 2 . There, the red and blue points correspond, respectively, to the slant ranges observed by to different beacons, $B_{1}$ and $B_{2}$. These ranges are originated from two different moving sources. It is clear from the Figure 2 that there is a continuous trend line, corresponding to the actual trajectories of the moving sources. Clutter measurements can also be observed, with a particular incidence by $B_{1}$, probably due to adverse location conditions. Such outlier points are expected and common when dealing with underwater acoustic signals. Because of that, the algorithms for estimating the position of the vehicles, whether for the tracking case or the navigation case, need to be robust, detecting and rejecting these outliers.

\section{AUV NAVIGATION}

AUVs require some sort of Navigation algorithm that is able to perform sensor fusion, combining data from different sensors, in order to provide the best possible estimate of

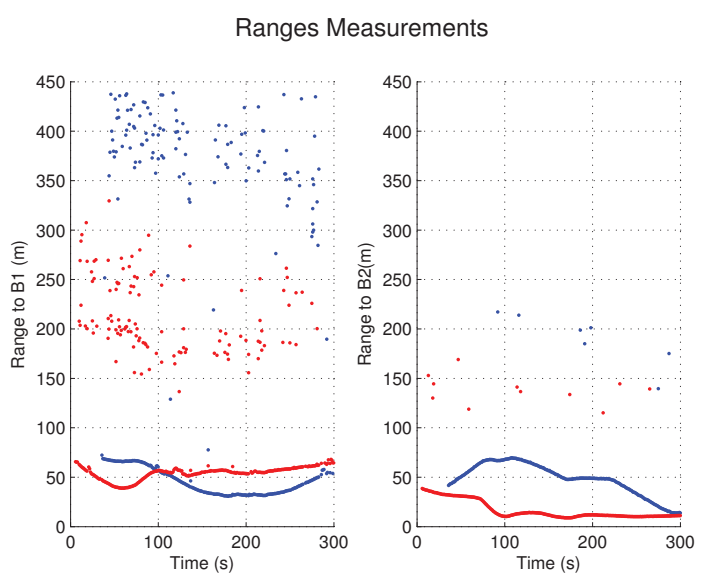

Fig. 2. Range measurements between two moving acoustic sources and two stationary beacons, $B_{1}$ and $B_{2}$. The different colours correspond to the different sources

the vehicle's position and velocity. The navigation algorithm, which is highly dependent on the vehicle and the sensors used, is usually based on an implementation of the Bayes Filter. In this section we will detail on a simple and generic navigation filter, based on an Extended Kalman Filter (EKF), that will be used for each of the moving vehicles. Thus, the focus in this section is not on obtaining the best possible navigation filter, but rather only on validating our proposal for the navigation of multiple vehicles, using OWTT range measurements. More advanced filters are likely to provide better results.

The navigation filter is responsible for propagating the vehicle's navigation equations, when available, include information from the different sensors. In our case the TOF of the signals, converted to the slant ranges between the vehicle and each of the acoustic beacons, will be used as indirect observations of the vehicle position and used to bound the error growth of the navigation equations. For the sake of simplicity we focus on the horizontal plane position only, but we are aware that depth sensors are already reliable enough to provide accurate depth measurements. A relatively simple state vector was chosen, $\mathbf{x}_{k}=\left[\begin{array}{llll}x_{k} & v_{x_{k}} & y_{k} & v_{y_{k}}\end{array}\right]^{T}$. Even though an augmented state vector would allow to accommodate data from other navigation sensor like IMUs, DVLs, or even model-based pseudo measurements, with a likely improve in the accuracy of the navigation solution, this wont be further pursued here. The discrete model used to predict the AUV motion is a simple constant velocity model, given by (1).

$$
\left[\begin{array}{c}
x_{k+1} \\
v_{x, k+1} \\
y_{k+1} \\
v_{y, k+1}
\end{array}\right]=\left[\begin{array}{cccc}
1 & \Delta_{t} & 0 & 0 \\
0 & 0 & 0 & 0 \\
0 & 0 & 1 & \Delta_{t} \\
0 & 0 & 0 & 0
\end{array}\right]\left[\begin{array}{c}
x_{k} \\
v_{x, k} \\
y_{k} \\
v_{y, k}
\end{array}\right]
$$

The received range measurements from each one of the acoustic beacons, $z_{k}$ are used to correct the Kalman Filter estimates that are propagated using the constant velocity model. To do so, we start by noting that the range measurements $r$, are a nonlinear function $g\left(\mathbf{x}_{k}\right)$ of the system state: 


$$
r=\sqrt{\left(x_{t}-x_{0, i}\right)^{2}\left(y_{t}-y_{0, i}\right)^{2}}
$$

Then, we compare $z_{k}$ with the expected range, $r^{*}$, which is calculated based on the current position estimates for the vehicles. Recalling from Figure 2, a high number of outliers are expected to be present in the received range measurements. Therefore, a strategy is implemented to prevent such spurious measurements to corrupt the behaviour of the filter. The first step is to consider, in each period of reception, only the first range provided by each one of the beacons. We are only interested on the the direct path of the signals, and we wish to discard the multiple reflections acoustic signals might have undergone. Nevertheless, some spurious measurements might still occur. An additional validation gate of the received ranges is also implemented according to

$$
\left(z_{k}-r^{*}\right)^{T} S_{k}^{-1}\left(z_{k}-r^{*}\right) \leq \gamma .
$$

Because the difference $\left(z_{k}-r^{*}\right)$ is assumed to be Gaussian, the value for $\gamma$ can be obtained from an appropriate $\chi^{2}$ distribution, for the desired confidence level. $S_{k}$ is the corresponding innovation covariance matrix, obtained as

$$
S_{k}=H_{k}^{T} P_{k} H_{k}+R_{k}
$$

where $H_{k}$ is the Jacobian of the measurement vector, $P_{k}$ the error covariance matrix and $R_{k}$ the covariance matrix of the observation noise.

A final note to the rate at which range measurements are received which is, on average, equal to the number of acoustic beacons per cycle of communications. Compared to standard LBL interrogation cycles, in our approach that rate is relatively higher, at least doubling the rate, which is a major advantage for the accuracy of the filter.

\section{Multiple AuV Tracker}

As previously mentioned above, the solution here presented for the simultaneous navigation and tracking of multiple vehicles requires all the vehicles need to synchronously emit similar acoustic signals. These signals will be detected by all the beacons and will enable the tracking of the vehicles. However, the main difficulty related with such approach is the fact that there is no explicit association between the signals detected by each of the beacons, and the vehicles. A suitable estimator must then be derived, in order to estimate the position of all the vehicles. Perhaps the biggest novelty in the approach we here propose is the multiple AUV tracker. The novelty consists on having a filter that is able to track multiple vehicles when the acoustic signals they emit are indistinguishable. Such AUV tracker, based on the Probability Hypothesis Density (PHD) Filter, is based on the previous work by the authors, where a Multiple AUV Tracker was envisioned [12].

The PHD Filter, initially proposed in [13], is an approximation of the general multitarget-multisource Bayes Filter. Contrary to standard Bayesian Filtering techniques, such approach uses Random Finite Sets (RFS) to model both the targets states and the measurement equations. The use of RFS overcomes the need for an explicit data association between targets and sources, which is found to be appropriate for the current application. On the other hand, the use of RFSs significantly alleviates the effort of addressing situations like target (dis)appearance and spawning, extended targets, false alarms and missed detections.

The PHD filter predictor equations are given by (5-6) while the PHD filter corrector equations are in (7-8). These equations are the core of the general PHD filter.

$$
\begin{aligned}
\mathbf{D}_{k \mid k-1}\left(\mathbf{X} \mid \mathbf{Z}^{k}\right)= & \gamma_{k}\left(\mathbf{x}_{k}\right)+ \\
& \int \phi_{k \mid k-1}\left(\mathbf{x}_{k-1}\right) \mathbf{D}_{k \mid k}\left(\mathbf{X} \mid \mathbf{Z}^{k}\right) d_{\mathbf{x}_{k-1}} \\
\phi_{k \mid k-1}\left(\mathbf{x}_{k-1}\right)= & p_{S, k}\left(\mathbf{x}_{k-1}\right) f_{k \mid k-1}\left(\mathbf{x}_{k} \mid \mathbf{x}_{k-1}\right)
\end{aligned}
$$

In the predictor equations the surviving target's density is propagated in time according to single target transition density $f_{k \mid k-1}$, and possible new-born targets are considered. $p_{S, k}$ refers to the probability a given target has to survive, from one time step the the immediately after, and $\gamma_{k}$ refers to the intensity of spontaneous births.

$$
\begin{gathered}
\mathbf{D}_{k \mid k} \cong \mathbf{L}_{\mathbf{Z}_{k+1}}(\mathbf{X}) \mathbf{D}_{k}\left(\mathbf{X} \mid \mathbf{Z}^{k}\right) \\
\mathbf{Z}_{k+1}=\left(1-p_{D}(\mathbf{X})\right)+ \\
\sum_{Z \in \mathbf{Z}_{k}} \frac{p_{D}(\mathbf{X}) L_{Z}(\mathbf{X})}{\lambda_{k} c_{k}(Z)+\int p_{D} L_{Z}(\mathbf{X}) \mathbf{D}_{k \mid k-1} d \mathbf{X}}
\end{gathered}
$$

In the measurement update equations above, the likelihood of all the measurements from the set of possible measurements are evaluated considering the current density function, $\mathbf{D}_{k \mid k}$. $c_{k}$ refers to the clutter spatial distribution and $\lambda_{k}$ to the clutter rate. Also, $p_{D}$ is the probability of detection of a target, and $L_{Z}$ the measurement likelihood. For our application, we choose to use a Sequential Monte Carlo (SMC) implementation of the PHD filter, particularly due to the simplicity to address the non-linearities present in the problem.

The single target state vector is defined to be $\mathbf{x}_{k}^{i}=$ $\left[\begin{array}{lll}x_{k}^{i} & y_{k}^{i} & 1_{k}^{i}\end{array}\right]$, where $x_{k}^{i}$ and $y_{k}^{i}$ refer to the position coordinates of the target while $l_{k}^{i}$ is the label used to identify each target. The use of a label allows for easy track labelling and simplifies the target estimation process. Naturally, the index $i$ refers to the target $i$, while $k$ refers to the time instant. Analogously to the navigation filter of the previous section, we chose a constant velocity model as the target motion model, but in here time velocity estimates are obtained through a Recursive Least Square (RLS) Estimator with forgetting factor, where the positions and times serve as an input. The measurement set $\mathbf{Z}_{k}$ is composed by the set of all the possible vectors $z_{k}^{i}=\left[\begin{array}{lll}r_{1} & \ldots & r_{n}\end{array}\right]^{T}$ that can be obtained from the observed ranges, where $r_{i}$ is the range measurement to acoustic beacon $B_{i}$, as given by (2). The likelihood of the measurements is 
considered to be Gaussian. For a large number of vehicles appropriate gating strategies must be derived in order to accommodate the increase in computational complexity.

\section{RESULTS}

In order to experimentally validate our approach, a series of tests were performed. For practical reasons, two independent experiments were performed in order to assess the performance of both the navigation and tracking algorithms separately. In this section we provide the details of these field trials and present the obtained results.

The use of AUVs poses a problem when it comes to establishing a ground-truth to be able to compare the results with. For that reason, on these trials we used Autonomous Surface Vehicles (ASVs) as AUV surrogates. The idea is that the ASVs can mimic the use of AUVs if they are equipped with an acoustic transducer that always remains underwater, and at a constant depth. By doing so we can have access to a set of acoustic underwater slant ranges obtained between a series of moving vehicles and the respective beacons and, but also the ground-truth data of the position of the vehicles, provided by the GPS receivers that equip the ASVs. The ASVs used can be seen in Figure 3.

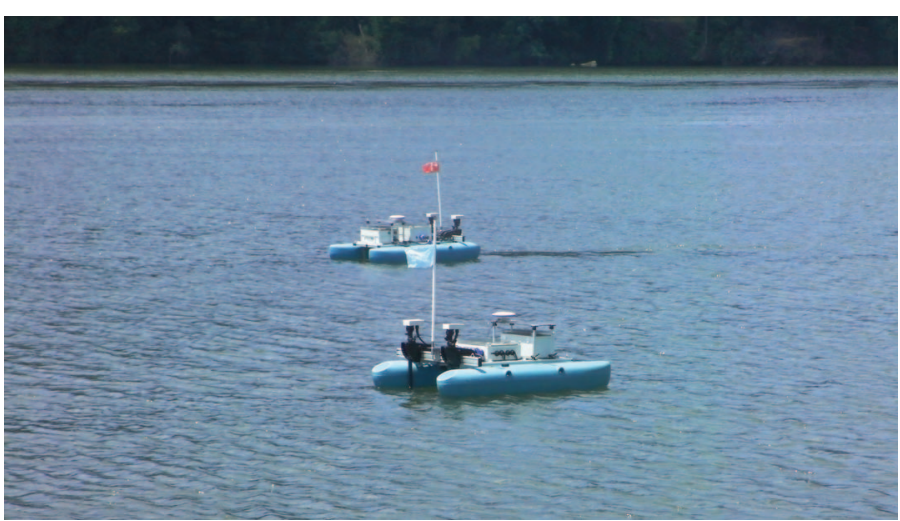

Fig. 3. The AUV surrogates used in the field trials

\section{A. Navigation}

In the first instance of the field trials we were interested on assessing the performance of the acoustic network while two vehicles were navigating simultaneously. For the sake of simplicity of the missions, we used two surface acoustic beacons and two AUV surrogates, one doing a station keeping manoeuvre and the other one moving from one specific location to another. The ranges obtained by each one of the vehicles can be seen in Figure 4. Besides the presence of some outliers (rejected ones in black), it can also be seen that Vehicle 1 has been affected by some shortage of range measurements, particularly visible between around the time instant $t=50 \mathrm{~s}$.

In Figure 5 it is possible to observer the trajectories of the vehicles, together with the position of the buoys. It is clear that even thought the trajectories obtained by the navigation filter do resemble the ground-truth data, there are still significant

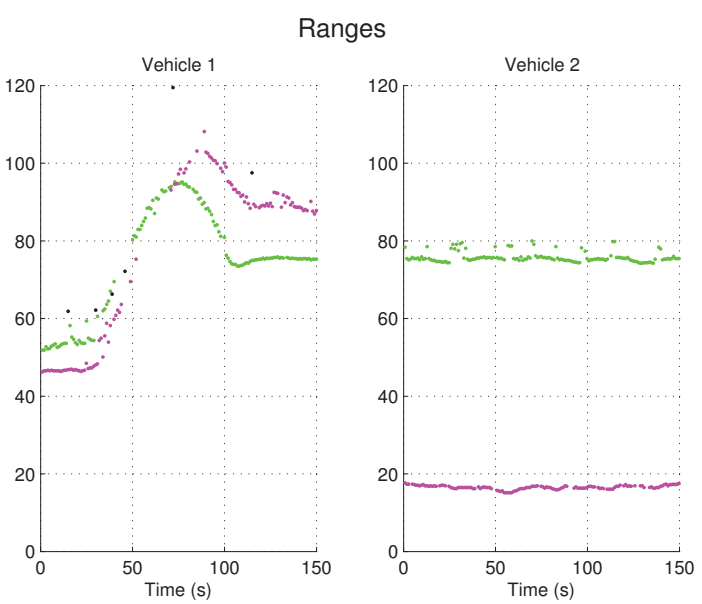

Fig. 4. Ranges detected by each one of the vehicles. Green colour indicates ranges to $B_{1}$ and purple colour ranges to $B_{2}$

differences. A cause for that is probably the shortage of ranges data that affected mainly Vehicle 1 , together with the very simple motion model in use and no other sensory data of the vehicles. Figure 6 provides a more detailed view on the position of the different vehicles along time, and comparing it to the ground-truth data. There, it is more easily observed the effect that the lack of ranges has cause one of the vehicles, in blue, to drift away. Finally, in Figure 7 we present the plot of the absolute error in position along the time. Once again we can see a peak in position error for one of the vehicles, at around $t=50 \mathrm{~s}$. Based on that data we arrive to a figure of an $4.1 \mathrm{~m}$ and $2.8 \mathrm{~m}$ RMS error for each of the vehicles which are, given the circumstances, very encouraging results.

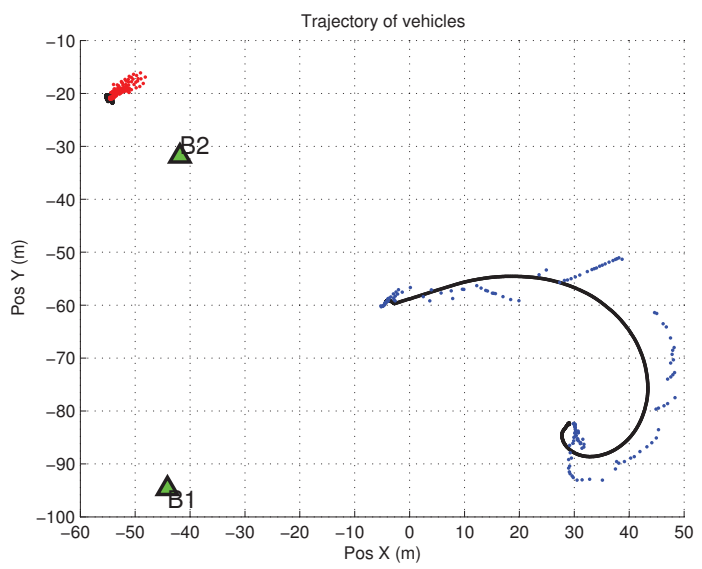

Fig. 5. Navigation Results: trajectories of the vehicles. In black ground-truth given by the GPS and in blue and red estimated by the navigation filter

\section{B. Tracking}

In the second instance of the field trials the goal was to assess the tracking results of the Multiple AUV Tracker, and a similar procedure was followed. The different parameters of the tracker used to obtain the results that are going to be presented are specified in Table I. 

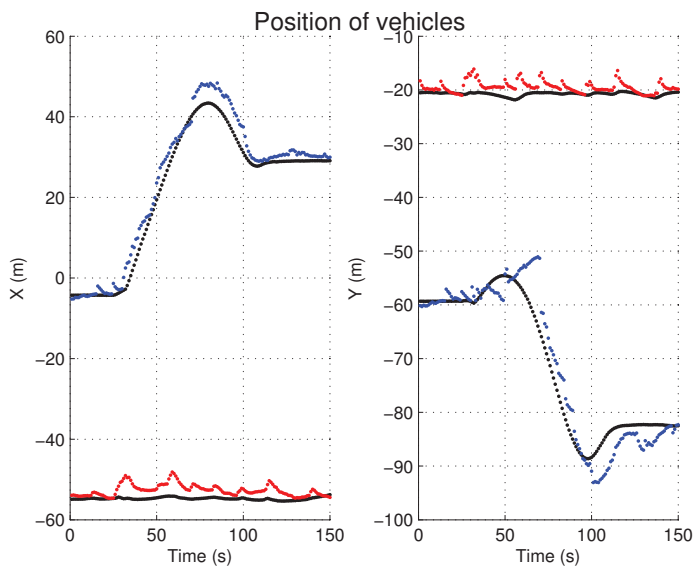

Fig. 6. Navigation Results: evolution of the position of the vehicles. In black ground-truth given by the GPS and in blue and red estimated by the navigation filter

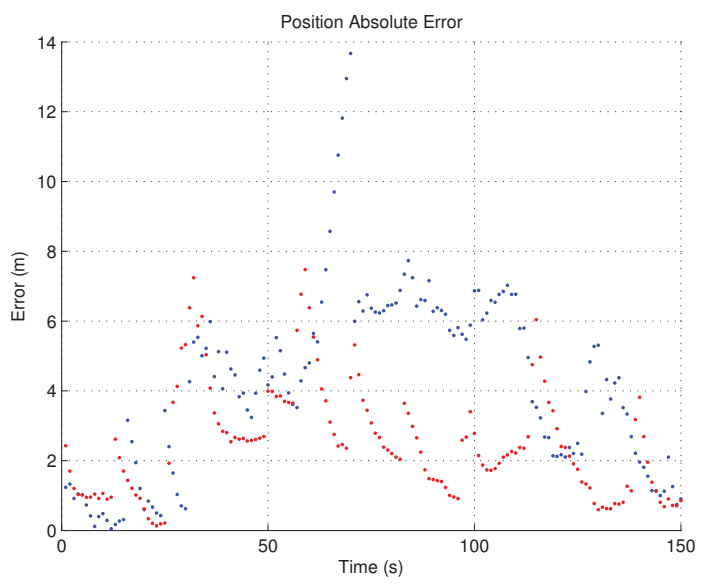

Fig. 7. Navigation Results: absolute position error for each of the vehicles, when comparing to ground truth given by GPS.

\begin{tabular}{|c|c|}
\hline Parameter & Value \\
\hline \multicolumn{2}{|l|}{ Filter Settings } \\
\hline Particles Per Target (N) & 1000 \\
\hline Particles Per Birth (M) & 1000 \\
\hline \multicolumn{2}{|l|}{ Predictor Settings } \\
\hline Prob. of Survival $\left(p_{s}\right)$ & 0.99 \\
\hline Prob. of Birth $\left(p_{b}\right)$ & 0.01 \\
\hline Process Noise Variance $\left(\sigma_{x, y}^{2}\right)$ & 0.1 \\
\hline \multicolumn{2}{|l|}{ Corrector Settings } \\
\hline Prob. of Detection $\left(p_{d}\right)$ & 0.6 \\
\hline Measurement Noise Variance $\left(\sigma_{r, B}^{2}\right)$ & 0.8 \\
\hline Clutter Intensity $\left(\lambda_{k}\right)$ & $5 \times 10^{-5} \sqrt{\left.\left|\mathbf{Z}_{k}\right|\right)}$ \\
\hline
\end{tabular}

PARAMETERS OF THE MUlTiPle AUV TraCKER
The mission consisted on having two vehicles navigating simultaneously in a predefined area, under surveillance of two moored acoustic buoys. We had the vehicles starting to emit acoustic signals at different times, in order to illustrate the ability of the filter to detect new vehicles entering the surveillance area. At the same time, the vehicles were navigating with arbitrary varying velocities and in different directions, including stationary situations. An overview of the trajectories performed by each of the vehicles can be seen in Figure 8 . Figure 9 provides a more detailed view on the position of the different vehicles along time, and comparing it to the ground-truth data. There, it is more easily observed that at around $t=130 \mathrm{~s}$ one of the vehicle is diverging from the actual position, and this is likely caused by the appearance of ghost targets induced by the measurements received. Finally, in Figure 10 we present the plot of the absolute error in position along the time. Once again we can see a peak in position error for Vehicle 1 just after $t=130 \mathrm{~s}$. Based on that data we arrive to a figure of an $2.1 \mathrm{~m}$ and $1.4 \mathrm{~m}$ RMS error, respectively, for both of the vehicles.

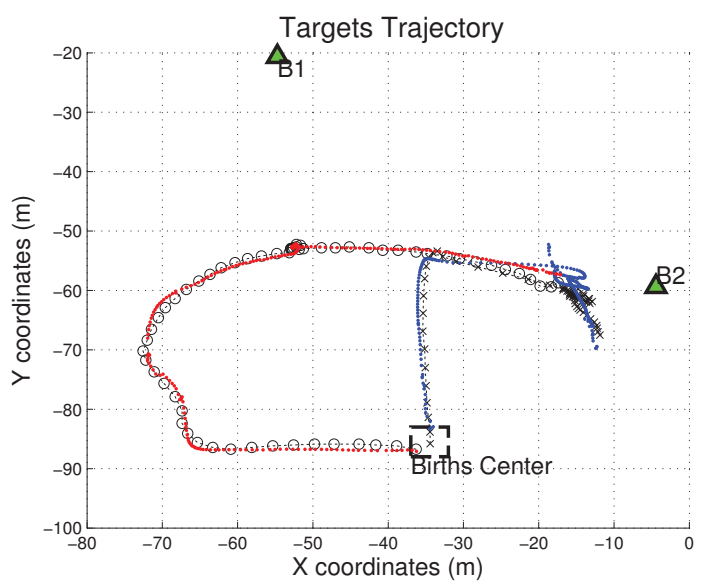

Fig. 8. Tracking results: trajectories of the vehicles. In black ground-truth given by the GPS and in blue and red estimated by the Multiple AUV Tracker.

\section{CONCLUSION}

This article is a preliminary work with the purpose of evaluating the feasibility of a novel LBL network that enables the navigation and external tracking of multiple vehicles. The approach we propose is composed out of three main components: the acoustic network itself, the navigation filters for each one of the vehicles, and the centralized tracker for multiple AUVs. Up to the authors knowledge, no similar approach has been previously proposed in the literature. The acoustic network is based on the use of a set of synchronized, GPS enabled, OWTT acoustic beacons, in the form of buoys that are surface deployed. Each of the beacons broadcast its own acoustic signals, that will help the navigation of a fleets of AUVs. On the vehicle side a navigation Filter based on EKF is responsible for fusing the selected range measurements with the general navigation solution of the vehicle. Finally, a centralized Multiple AUV Tracker based 

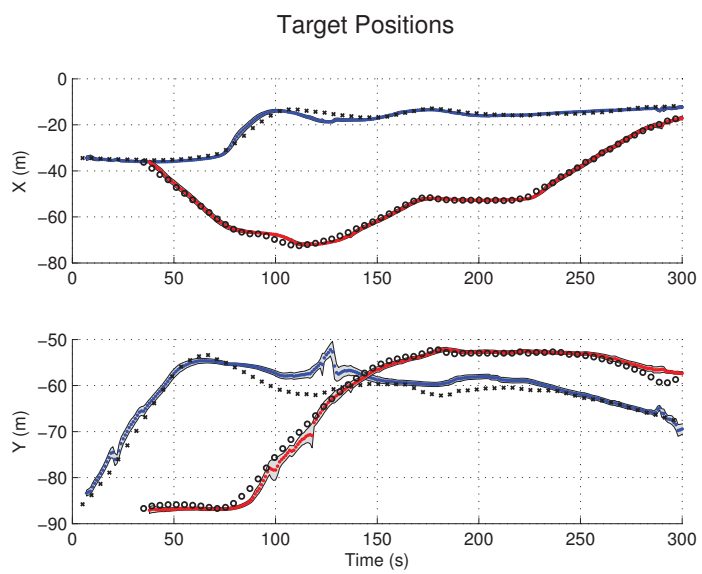

Fig. 9. Tracking Results: evolution of the position of the vehicles. In black ground-truth given by the GPS and in blue and red estimated by the Multiple AUV Tracker.

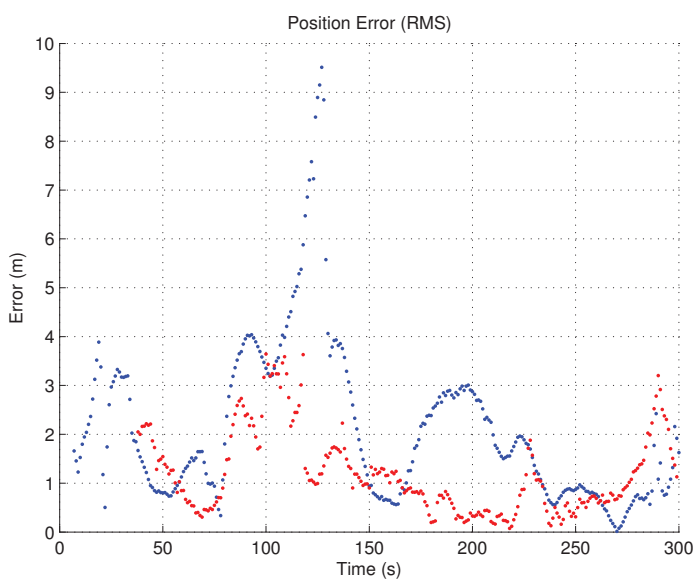

Fig. 10. Tracking Results: absolute position error for each of the vehicles, when comparing to ground truth given by GPS.

on a PHD filter collects the signals that all the beacons are able to detect, and is then responsible to estimate the position of each the vehicles. Experimental evidence on the success of the proposed approach was provided. Nevertheless, further testing the overall solution in a single test instance is necessary to assess its robustness. Future work will also include robustifiyng the proposed solution, for example by developing appropriate measurement gating strategies.

\section{ACKNOWLEDGMENT}

This work is financed by the ERDF - European Regional Development Fund through the Operational Programme for Competitiveness and Internationalisation - COMPETE 2020 Programme within project «POCI-01-0145-FEDER-006961», and by National Funds through the FCT - Fundação para a Ciência e a Tecnologia (Portuguese Foundation for Science and Technology) as part of project UID/EEA/50014/2013. The first author was supported by the Portuguese Foundation for Science and Technology through the Ph.D. grant «SFRH / BD / 70727 / 2010».

\section{REFERENCES}

[1] L. Paull, S. Saeedi, M. Seto, and H. Li, "Auv navigation and localization: A review," Oceanic Engineering, IEEE Journal of, vol. 39, no. 1, pp. 131-149, Jan 2014.

[2] J. Melo and A. Matos, "Guidance and control of an asv in auv tracking operations," in OCEANS 2008, Sept 2008, pp. 1-7.

[3] A. Matos and N. Cruz, "Simultaneous acoustic navigation of multiple auvs, 2006," in Proceedings of the Conference MCMC - Maneuvering and Control of Marine Crafts, Lisbon, September 2006.

[4] B. Baker, D. Odell, M. Anderson, T. Bean, and D. Edwards, "A new procedure for simultaneous navigation of multiple auv's," in OCEANS, 2005. Proceedings of MTS/IEEE, Sept 2005, pp. 1-4.

[5] N. Kottege and U. R. Zimmer, "Underwater acoustic localization for small submersibles," Journal of Field Robotics, vol. 28, no. 1, pp. 4069, 2011.

[6] M. Fallon, G. Papadopoulos, and J. Leonard, "A measurement distribution framework for cooperative navigation using multiple auvs," in Robotics and Automation (ICRA), 2010 IEEE International Conference on, May 2010, pp. 4256-4263.

[7] R. Eustice, L. Whitcomb, H. Singh, and M. Grund, "Experimental results in synchronous-clock one-way-travel-time acoustic navigation for autonomous underwater vehicles," in Robotics and Automation, 2007 IEEE International Conference on, Apr. 2007, pp. 4257-4264.

[8] R. M. Eustice, H. Singh, and L. L. Whitcomb, "Synchronous-clock oneway-travel-time acoustic navigation for underwater vehicles," Journal of Field Robotics, Special Issue on State of the Art in Maritime Autonomous Surface and Underwater Vehicles, vol. 28, no. 1, pp. 121-136, 2011.

[9] S. E. Webster, R. M. Eustice, H. Singh, and L. L. Whitcomb, "Advances in single-beacon one-way-travel-time acoustic navigation for underwater vehicles," The International Journal of Robotics Research, vol. 31, no. 8 pp. 935-950, 2012.

[10] S. Webster, J. Walls, L. Whitcomb, and R. Eustice, "Decentralized extended information filter for single-beacon cooperative acoustic navigation: Theory and experiments," Robotics, IEEE Transactions on, vol. 29, no. 4, pp. 957-974, Aug 2013.

[11] P. Batista, "Long baseline navigation with clock offset estimation and discrete-time measurements," Control Engineering Practice, vol. 35, pp. $43-53,2015$.

[12] J. Melo and A. Matos, "A phd filter for tracking multiple auvs," in Oceans - St. John's, 2014, Sept 2014, pp. 1-8.

[13] R. Mahler, "Multitarget bayes filtering via first-order multitarget moments," Aerospace and Electronic Systems, IEEE Transactions on, vol. 39, no. 4, pp. 1152-1178, Oct 2003. 